\title{
Campylobacter biotyping scheme of epidemiological
} value

\author{
FJ BOLTON, AV HOLT, DN HUTCHINSON
}

From the Public Health Laboratory, Preston Infirmary, Preston, Lancashire PR1 6RS

SUMMARY A biotyping scheme has been developed which utilises 12 tests, including growth at $28^{\circ} \mathrm{C}$, hippurate hydrolysis, and 10 resistotyping tests. These tests are arranged in groups of three, and by assigning a numerical value to each positive test a four figure code is produced for each strain. The order of the tests is such that campylobacters are both speciated and biotyped. This scheme recognises Campylobacter jejuni, $C$ coli, " $C$ laridis," $C$ fetus fetus, and $C$ fetus subspecies venerealis. The reproducibility of the biotyping technique and the stability of the biotype code have been determined by testing campylobacter reference strains. The routine application of the scheme has also been evaluated by biotyping 1000 recent campylobacter isolates, and the epidemiological value has been confirmed by testing serotyped isolates from several milk borne outbreaks.

Isolation of Campylobacter jejuni is now common place, but the epidemiology of campylobacter enteritis has not been fully elucidated. This can be achieved only by detecting markers which differentiate strains from each other. Several of the laboratory techniques traditionally of epidemiological value have been investigated. Bacteriocine typing of campylobacters has been attempted,' but to date it has not proved useful. Phage typing, which is still at a developmental stage, may be more promising. ${ }^{2}$ The detection of plasmids ${ }^{3}$ and enzymatic profiles ${ }^{4}$ as epidemiological markers has also been suggested. Currently, two techniques, serotyping and biotyping, have been adopted by many workers and several systems have been reported.

There are two major serological typing schemes: one utilising heat stable antigens detected by a passive haemagglutination technique ${ }^{5}$ and the other based on heat labile antigens detected by slide agglutination. ${ }^{6}$ Although serotyping separates strains, it does not necessarily confirm identity of strains belonging to the same serotype. Biotyping of campylobacters has been available for several years; Skirrow and Benjamin ${ }^{78}$ described several tests which are useful for biotyping, and their shortened scheme $^{8}$ has become widely accepted. Two other biotyping schemes have recently been reportedone from the USA by Hebert $e t a l^{4}$ and the other by Lior $^{10}$ from Canada.
Since campylobacters are relatively inactive in biochemical tests we investigated the possible value of resistotyping these organisms. We have determined the susceptibility of Campylobacter spp to a selection of antibiotics, dyes, and chemicals in a search to find a set of differential tests. We have modified some tests described by other workers ${ }^{78} 11$ and included several new tests. This scheme employs twelve tests, including growth at $28^{\circ} \mathrm{C}$, hippurate hydrolysis $^{8 / 2}$ and 10 resistotyping tests. With the exception of the hippurate test all tests are performed in a blood free basal medium.

The scheme which speciates and biotypes campylobacters has been evaluated by testing campylobacter reference strains, recent isolates, and strains from several milk borne outbreaks. The results of these studies show the potential epidemiological value of the scheme.

\section{Material and methods}

MATERIAL

Blood free basal medium

Modified charcoal, ferrous, pyruvate (CFP) agar ${ }^{13}$ was prepared by adding the following ingredients to 1 litre of deionised water: nutrient broth no 2 (Oxoid) $25 \mathrm{~g}$, New Zealand agar (Davis Gelatine, NZ Ltd, Christchurch, New Zealand) $20 \mathrm{~g}$, bacteriological charcoal (Oxoid) $4 \mathrm{~g}$, casein hydrolysates (Oxoid) $3 \mathrm{~g}$. Five millilitres of $5 \%$ aqueous ferrous sulphate and $5 \mathrm{ml}$ of $5 \%$ aqueous sodium pyruvate were auded to give final concentrations for 
each of $0.025 \%$. The medium was then adjusted to $\mathrm{pH} 7.4$ and sterilised by autoclaving at $121^{\circ} \mathrm{C}$ for 15 min.

\section{Control organisms}

The following organisms were used: $C$ jejuni biotype $1^{8}$ NCTC 11168; $C$ jejuni biotype $2^{8}$ NCTC 11392 ; $C$ coli NCTC 11353; $C$ laridis $^{14}$ NCTC 11352; $C$ fetus fetus NCTC 5850.

These organisms were maintained at $-70^{\circ} \mathrm{C}$ in brain heart infusion broth (Difco) containing 20\% glycerol.

\section{Microaerobic atmosphere}

This was achieved by evacuating anaerobic jars without catalyst to $500 \mathrm{~mm} \mathrm{Hg}$ and replacing with $10 \%$ carbon dioxide in nitrogen.

\section{BIOTYPING TESTS \\ Preparation of inoculum}

Pure cultures of control and test organisms were obtained by subculture on to plates of the modified CFP agar, which were then incubated microaerobically at $37^{\circ} \mathrm{C}$ for $48 \mathrm{~h}$. Discrete colonies were then resubcultured on to modified CFP agar containing $1.2 \%$ agar and incubated as previously. Bacteria were harvested from these plates into $5 \mathrm{ml}$ of $0.1 \%$ peptone water and the suspensions adjusted to a standard opacity. This was done by preparing a suspension of the $C$ jejuni NCTC 11168, which was standardised to a density of about $2 \times 10^{*}$ colony forming units $(\mathrm{cfu} / \mathrm{ml})$ using a Perkin Elmer $6 / 20$ spectrophotometer at a wavelength of $450 \mathrm{~nm}$. All other suspensions were adjusted by visual reference to this freshly prepared standard.

\section{Hippurate hydrolysis}

This was performed as a micro-method. A $1 \%$ solution of sodium hippurate was prepared in phosphate buffer as suggested by Piot $e t a^{15}$ and $100 \mu \mathrm{l}$ dispensed into wells of flat bottomed microtitre plates.
A dense suspension of campylobacters was made in the substrate/buffer reagent by removing sufficient growth from purity plates with sterile wooden applicator sticks. Inoculated microtitre plates were incubated at $37^{\circ} \mathrm{C}$ for $2 \mathrm{~h}, 40 \mu \mathrm{l}$ of ninhydrin re-O agent ${ }^{15}$ added, reincubated at $37^{\circ} \mathrm{C}$ for $10 \mathrm{~min}$, and $\bar{O}$ then examined for the development of a purple $\overline{\bar{\rho}}$. colour, which was indicative of a positive result. ${ }^{\infty}$ Doubtful results were repeated using a tube test. ${ }^{15}$

\section{Temperature tolerance tests}

Plates of modified CFP agar were inoculated from $\overrightarrow{\vec{H}}$ the prepared suspension with sterile cotton tipped $\stackrel{\sigma}{\omega}$ swabs. Ten campylobacter suspensions were tested on each plate by streaking five suspensions on each $\dot{\omega}$ half of the plate. Tests were incubated microaerobi-. cally at $28^{\circ} \mathrm{C}$ for five days and at $37^{\circ} \mathrm{C}$ for $48 \mathrm{~h}$. (growth control). An aerobic plate incubated at $\forall$ $37^{\circ} \mathrm{C}$ for $48 \mathrm{~h}$ was included as a purity control.

\section{Resistotyping tests}

A list of the resistotyping reagents, their stock concentrations and storage conditions, and the final $\vec{\oplus}$ concentrations incorporated into the test plates is $\stackrel{\oplus}{\oplus}$ given in Table 1 . Test plates were prepared by: adding $18 \mathrm{ml}$ of cooled, molten modified CFP agar to petri dishes containing $2 \mathrm{ml}$ of 10 times concentrated resistotyping reagent. These were mixed, $\bar{\partial}$ allowed to set, and dried at room temperature overnight. Plates were inoculated by the method $\mathscr{Q}$ described for the temperature tolerance tests and $\overrightarrow{\vec{O}}$ incubated microaerobically at $37^{\circ} \mathrm{C}$ for $48 \mathrm{~h} .3$ Growth on the test plates was compared with growth on the modified CFP agar control, and resistotyping? plates showing more than $50 \%$ of growth obtained on the control plate were recorded as positive.

Calculation and interpretation of the biotype code number The twelve tests are arranged in four groups of three tests. Each positive result is given a numerical

Table 1 Resistotyping reagents

\begin{tabular}{|c|c|c|}
\hline Reagents & Stock concentration and storage conditions & Final concentration in modified CFP agar \\
\hline $\begin{array}{l}\text { Pyronin Y (Sigma)* } \\
\text { Safranin } 0 \text { (Sigma)* } \\
\text { Sodium arsenite (BDH)* } \\
\text { Potassium permanganate (BDH)* } \\
2-3-5 \text { triphenyl-tetrazolium chloride (BDH) } \\
\text { Cephazolin (Eli Lilly) } \\
\text { Metronidazole (May and Baker) } \\
\text { D-Cycloserine (Sigma) } \\
\text { 5-Fluorouracil (Sigma) } \\
\text { Nalidixic acid (Sterling Winthrop) } \ddagger\end{array}$ & $\begin{array}{l}0.2 \% \text { room temperature } \\
0.5 \% \text { room temperature } \\
0.01 \% \text { room temperature } \\
1 \% \text { room temperature } \\
0.5 \% \text { fresh } \dagger \\
20000 \mathrm{mg} / \mathrm{l}-20^{\circ} \mathrm{C} \\
500 \mathrm{mg} / 1-20^{\circ} \mathrm{C} \\
600 \mathrm{mg} / \mathrm{l}-20^{\circ} \mathrm{C} \\
10000 \mathrm{mg} / \mathrm{l}-20^{\circ} \mathrm{C} \\
6000 \mathrm{mg} / \mathrm{l}-20^{\circ} \mathrm{C}\end{array}$ & $\begin{array}{l}0.02 \% \\
0.05 \% \\
0.001 \% \\
0.1 \% \\
0.05 \% \\
200 \mathrm{mg} / 1 \\
5 \mathrm{mg} / 1 \\
6 \mathrm{mg} / 1 \\
100 \mathrm{mg} / \mathrm{l} \\
60 \mathrm{mg} / \mathrm{l}\end{array}$ \\
\hline \multicolumn{3}{|c|}{$\begin{array}{l}\text { All stock concentrations were prepared in sterile distilled water. } \\
\text { *These reagents were sterilised at } 115^{\circ} \mathrm{C} \text { for } 10 \mathrm{~min} \text {. } \\
\text { †Solution kept in the dark until required. } \\
\ddagger \text { Dissolved initially in } 0.5 \mathrm{~N} \mathrm{NaOH} \text {. }\end{array}$} \\
\hline
\end{tabular}


value-that is, in any triplicate of tests if test $A$ is positive score 1 , if test $B$ is positive score 2 , and if test $C$ is positive score 4 . The sum of the positive results is then calculated for each group of tests-for example, if test $\mathbf{A}$ is negative score 0 , if test $B$ is positive score 2 , and if test $C$ is positive score 4 . This gives a total score of 6 . This system when applied to all four groups gives a four number code.

The first three tests are arranged to determine the species of campylobacter-for example $C$ jejuni codes $6 \ldots \ldots, C$ coli codes $2_{-} \ldots, C$ fetus codes 1 --- , and $C$ laridis codes $0---$ The remaining which can be used for epidemiological purposes.

Assessment of the reproducibility and stability of the biotyping scheme

For these studies 13 campylobacter NCTC reference strains and $25 C$ fetus strains were tested. To determine the reproducibility of the biotype code, these strains were tested on three separate occasions, after repeated subculture on to modified CFP agar. Stability of the biotype code was determined by retesting these strains after storage at $-70^{\circ} \mathrm{C}$ for at least one month.

\section{Biotyping of recent campylobacter isolates}

About 1000 campylobacter isolates from man, chickens, cattle, sheep, pigs, offal, milk, and water were tested by the biotyping scheme over an 18 month period.

\section{Biotyping of campylobacters from outbreaks}

To determine the epidemiological value of the biotyping scheme we tested strains from five milk borne outbreaks. All of the strains had been serotyped by Dr DM Jones, Public Health Laboratory, Manchester, England, using the Penner serotyping scheme. ${ }^{5}$

\section{Results}

\section{REPRODUCIBILITY AND STABILITY OF BIOTYPE} CODE

The reference strains and the $C$ fetus strains tested on several occasions after serial subculture gave the same biotype codes. Similarily, when these campylobacters were retested after storage at $-70^{\circ} \mathrm{C}$ the biotype profile was identical. The biotype code numbers for the reference strains and the $C$ fetus strains are shown in Tables 2 and 3 respectively. Biotyping of $C$ jejuni, $C$ coli, and $C$ laridis reference strains indicated that different biotypes are present within each species.

\section{BIOTYPING OF RECENT ISOLATES}

Three different Campylobacter spp were recognised
Table 2 Biotype codes of campylobacter reference strains

\begin{tabular}{lll}
\hline Campylobacter spp & NCTC number & Preston biotype code \\
\hline C jejuni & 11322 & 6012 \\
& 10983 & 6110 \\
& 11168 & 6112 \\
& 11385 & 6150 \\
c coli & 11392 & 6264 \\
& 11350,11353 & 2724 \\
C laridis & 11438 & 2734 \\
C fetus fetus & 11437 & 2774 \\
\hline
\end{tabular}

Table 3 Biotype codes of $C$ fetus strains

\begin{tabular}{lll}
\hline Campylobacter spp & No of strains & Preston biotype code \\
\hline C fetus fetus & 2 & 1125 \\
(intestinalis) & 1 & 1131 \\
& 1 & 1135 \\
C fetus venerealis & 9 & 1137 \\
C fetus venerealis & 1 & 1001 \\
intermedius & 1 & 1001 \\
& 1 & 1121 \\
& 1 & 1131 \\
\hline
\end{tabular}

from the 1000 recent isolates. These comprised 779 strains of $C$ jejuni, 183 strains of $C$ coli, and 38 strains of $C$ laridis.

Table 4 shows the percentage positive results given by campylobacters for each of the biotyping tests. The first three tests distinguish between the three Campylobacter spp. Although the second, third, and fourth group of tests are important determinants of the biotype profile, some of the tests show trends which are useful confirmatory characteristics of the different Campylobacter spp. C laridis strains are always negative in the second group of tests and, although this helps to confirm the identity of these strains, it should be noted that about $14 \%$ of $C$ jejuni and $5 \%$ of $C$ coli strains can give negative results in these three tests. Resistance to pyronin is associated with $70 \%$ of $C$ coli strains and this test is therefore a useful indicator for the hippurate negative species.

In the third group of tests resistance to metronidazole and safranin is most commonly found in $C$ laridis strains and when combined with other tests can be useful for recognising atypical $C$ laridis strains. The fourth group of tests includes resistance to nalidixic acid, which is mainly associated with $C$ laridis strains, but we have found some strains which are sensitive. In contrast, we have found a few $C$ jejuni strains which are resistant to nalidixic acid. All $C$ coli strains tested so far have been sensitive. Resistance to 5-fluorouracil is also more common in $C$ laridis strains. Resistance to cyloserine is a useful confirmatory test for differentiating between $C$ jejuni, which are mainly sensitive, and $C$ coli and $C$ laridis, which are usually resistant. 
Table 4 Biotyping of 1000 campylobacter isolates

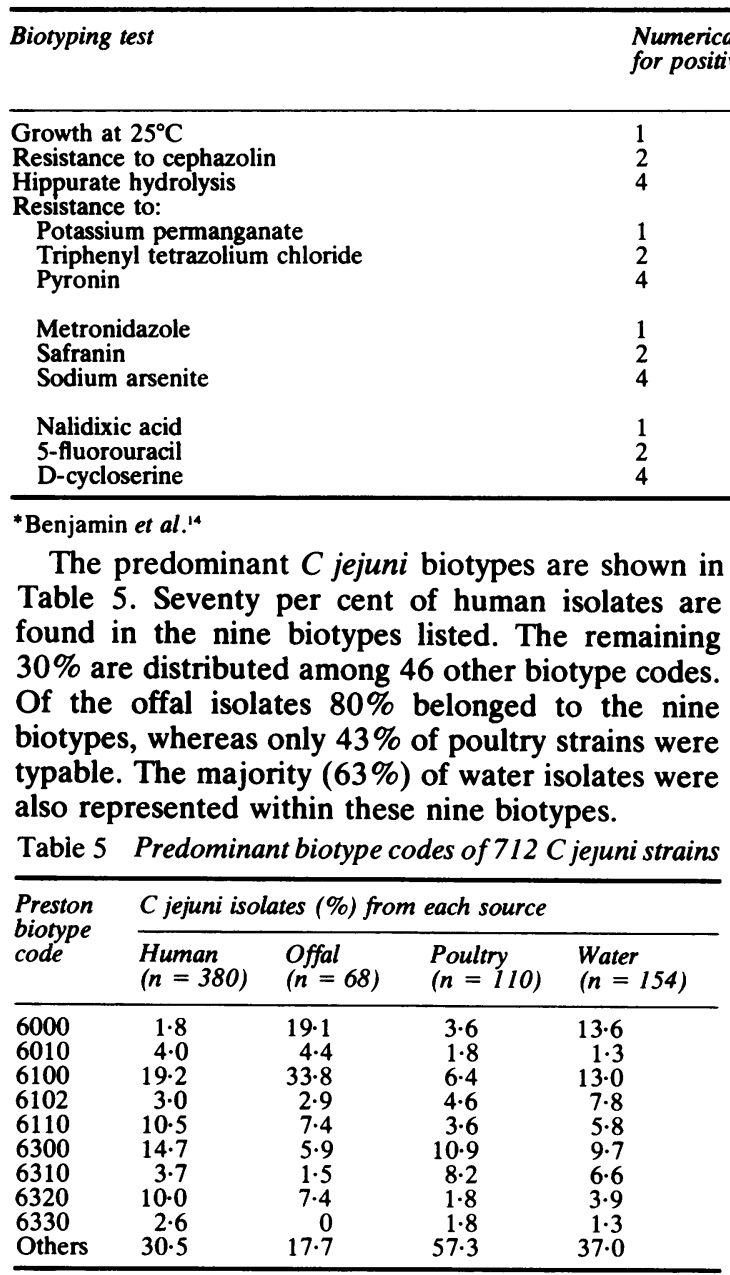

BIOTYPING OF OUTBREAK STRAINS

Table 6 shows the serotypes and biotypes of $C$ jejuni isolates from the five outbreaks.

Although $C$ jejuni serotype 4 was isolated from both the Leeds and York outbreaks, the biotype codes were different. Similarly, isolates from the Pennington and Arnside outbreaks were $C$ jejuni serotype 2, but were also different biotypes.

Eight human and four cattle isolates from the Arnside outbreak were $C$ jejuni serotype 2 and were identical biotypes. Two other cattle isolates, however, which were not serotypable were shown to be different biotypes.

\section{Discussion}

This biotyping scheme was developed for epidemiological purposes. It is a relatively easy

Table 6 Biotyping of campylobacters from milkborne outbreaks

\begin{tabular}{llcc}
\hline $\begin{array}{l}\text { Outbreak } \\
\text { location }\end{array}$ & $\begin{array}{l}\text { No of strains } \\
\text { tested }\end{array}$ & Penner serotype Preston biotype \\
code
\end{tabular}

${ }^{*}$ Human faecal samples.

†Cattle rectal swabs.

NT $=$ non-typable.

technique to establish and is within the capabilities $\overrightarrow{\vec{O}}$ of most laboratories but will appeal primarily to those who are unable to obtain a comprehensive range of antisera or are not fortunate enough to be able to raise their own antisera. It has the advantage over serology that every strain can be ascribed a:biotype code. Presently not all strains are serotypable using antisera to the heat stable and labile antigens.

Included in the scheme are tests which determineo resistance to antibiotics and chemicals, a property which may be plasmid mediated. As yet we have noto investigated the phenomenon, but to date we have not found active loss or acquisition of resistance. Both test and control strains have been repeatedly $N^{\circ}$ subcultured and when biotyped consistently give the N same code. Also test and reference strains have beeno stored at $-70^{\circ} \mathrm{C}$ for varying periods ranging from aे few weeks to a year and on recovery have retained the initial biotype pattern. We are confident that the method is reproducible and that the biotype code of the campylobacter organisms is stable.

The results obtained with the reference strains (Table 2) shows that the arrangement of the testso speciates the strain being examined. The chemicalso and antibiotics selected and their groupings were 
primarily made to enable us to type the thermotolerant campylobacters associated with human infections. It is with interest that we present the results of examining strains of $C$ fetus (Table 3 ). On the basis of our biotyping code three of the four strains of $C$ fetus venerealis intermedius would be speciated as $C$ fetus fetus and the fourth as $C$ fetus venerealis. We believe that this biotyping system will be of value to veterinary workers, who regularly isolate these organisms especially as $C$ fetus fetus can be differentiated into several biotype codes.

Comparison of the biotype codes of strains isolated from humans with the isolates from animal and other environmental sources (Table 5) shows that the biotypes of human strains are found in both offal and poultry, though a particular code may predominate in either animal source. An example is biotype code 6100 , which is the commonest human biotype and is found in one third of offal strains but in only $6.4 \%$ of isolates from poultry. Likewise, biotype codes 6300 and 6310 form $19 \cdot 1 \%$ of poultry isolates and only $7.4 \%$ of offal strains and yet comprise $18.4 \%$ of campylobacters isolated from human sources.

Several schemes for biotyping thermotolerant campylobacters have been developed. ${ }^{7-10}$ These systems use between three and five tests and if used alone the epidemiological information achieved is limited. Moreover, $C$ jejuni biotype 1 of one group of workers may not be the same as $C$ jejuni biotype 1 of a different group. The biotype code used in this study, being a four figure code, avoids direct confusion with existing numbering. Nevertheless, we believe that for maximal epidemiological information two different but complementary systems are required. The potential value of combining a serological and biotyping system is shown by the results given in Table 6 . Lior $^{10}$ has recently described an extended biotyping scheme which he believes should be used in conjunction with his serotyping scheme.

We have used our biotyping scheme during the past year to investigate several outbreaks and incidents of campylobacter infection and have found it capable of providing epidemiological information comparable to that provided by present serological methods. Its evaluation with serological tests is continuing and will form the basis of further publication.

We thank Dr DM Jones and Mrs EM Sutcliffe for providing the serotyped strains of campylobacters from the milk borne outbreaks; Dr RJ Owen, National Collection of Type Cultures, Colindale, for the campylobacter reference strains; KPW Gill and KP Lander, Central Veterinary Laboratory, Wey- bridge; SD Neill, Veterinary Research Laboratories, Belfast, for the $C$ fetus strains; and Mrs MC May for typing the manuscript.

\section{References}

1 Telfer Brunton WA, Moyes A. The use of Pseudomonas aeruginosa for the typing of thermophilic campylobacters. In: Newell DG, ed. Campylobacter: epidemiology, pathogenesis and biochemistry. Lancaster: MTP, 1982:60-1.

${ }^{2}$ Ritchie AE, Bryner JH, Foley JW. Phages of thermophilic Campylobacter spp.: isolation, morphology and utility for typing. In: Pearson AD, Skirrow MB, Rowe B; Davies J, Jones DM, eds. Campylobacter II. Proceedings of the second international workshop of campylobacter infections. London: Public Health Laboratory Service, 1983:101-2.

${ }^{3}$ Ambrosio RE, Lastovica AJ. Rapid screening procedure for detection of plasmids in campylobacters. In: Pearson AD, Skirrow MB, Rowe B, Davies J, Jones DM, eds. Campylobacter II. Proceedings of the second international workshop of campylobacter infections. London: Public Health Laboratory Service, 1983:28.

4 Elharrif Z, Megraud F. Enzymatic profiles of thermophilic campylobacters. In: Pearson AD, Skirrow MB, Rowe B, Davies J, Jones DM, eds. Campylobacter II. Proceedings of the second international workshop of campylobacter infections. London: Public Health Laboratory Service, 1983:44.

${ }^{5}$ Penner JL, Hennessy JN. Passive haemagglutination technique for serotyping Campylobacter fetus subsp jejuni on the basis of soluble heat-stable antigens. J Clin Microbiol 1980;12:732-7.

${ }^{6}$ Lior H, Woodward DL, Edgar JA, Laroche LJ, Gill P. Serotyping of Campylobacter jejuni by slide agglutination based on heat-labile antigenic factors. J Clin Microbiol 1982;15:761-8.

' Skirrow MB, Benjamin J. '1001' Campylobacters: cultural characteristics of intestinal campylobacters from man and animals. J Hyg 1980; 85:427-42.

skirrow MB, Benjamin J. Differentiation of enteropathogenic campylobacter. J Clin Pathol 1980;33:1122.

${ }^{9}$ Hebert GA, Hollis DG, Weaver RE, Lambert MA, Blaser MJ, Moss CW. 30 years of campylobacters: biochemical characteristics and a biotyping proposal for Campylobacter jejuni. $J$ Clin Microbiol 1982; 15:1065-73.

${ }^{10}$ Lior H. A new extended biotyping scheme for Campylobacter jejuni, $C$ coli and "C laridis" (NARTC). In: Pearson AD, Skirrow MB. Rowe B, Davies J, Jones DM, eds. Campylobacter II. Proceedings of the second international workshop of campylobacter infections. London: Public Health Laboratory Service, 1983:42.

"Karmali MA, De Grandis S, Fleming PC. Antimicrobial susceptibility of Campylobacter jejuni and Campylobacter fetus subsp fetus to eight cephalosporins with special reference to species differentiation. Antimicrob Agents Chemother 1980;18:94851.

${ }^{12}$ Harvey SM. Hippurate hydrolysis by Campylobacter fetus. J Clin Microbiol 1980;11:435-7.

${ }^{13}$ Bolton FJ, Coates D. Development of a blood-free campylobacter medium: screening tests on basal medium and supplements and the ability of selected supplements to facilitate aerotolerance. J Appl Bacteriol 1983;54:115-25.

14 Benjamin J, Leaper S, Owen RJ, Skirrow MB. Description of Campylobacter laridis, a new species comprising the nalidixic acid resistant thermophilic campylobacter (NARTC) group. Curr Microbiol 1983;8:231-8.

${ }^{15}$ Piot P, Van Dyck E, Totten PA, Holmes KK. Identification of Gardnerella (Haemophilus) Vaginalis. J Clin Microbiol 1982;15: 19-24.

Requests for reprints to: Mr FJ Bolton, Public Health Laboratory, Royal Infirmary, Meadow Street, Preston PR1 6PS, England. 